\title{
A RARE CASE OF CARPAL MALORHEOSTOSIS
}

Pavithra Sangarikonda ${ }^{1}$, Kotha Vamshi Krishna ${ }^{2}$, Bhuvana Nuthalapati ${ }^{3}$ Tatikonda Venkat Kishan ${ }^{4}$, (Brig) R. S. Moorthy5.

1. Junior Resident, Department of Radiology, Kamineni Institute of Medical Sciences.

2. Assistant Professor, Department of Radiology, Kamineni Institute of Medical Sciences.

3. Junior Resident, Department of Radiology, Kamineni Institute of Medical Sciences..

4. Senior Resident, Department of Radiology, Kamineni Institute of Medical Sciences.

5. Professor and HOD, Department of Radiology, Kamineni Institute of Medical Sciences.

\section{CORRESPONDING AUTHOR}

\section{Dr. (Brig) R. S. Moorthy,}

Prof\&HOD Radiodiagnosis,

Kamineni Institute of Medical Sciences,

Narketpally, 508254,

Nalagonda dist,Andhra Pradesh.

Email-dr_rsatyam1@yahoo.co.in

\section{HOW TO CITE THIS ARTICLE:}

Pavithra Sangarikonda, Kotha Vamshi Krishna, Bhuvana Nuthalapati, Tatikonda Venkat Kishan, (Brig) R. S. Moorthy. "A Rare Case of Carpal Malorheostosis". Journal of Evolution of Medical and Dental Sciences 2013; Vol2, Issue 24, June 17; Page: 4410-4413.

ABSTRACT: Melorheostosis is a rare, non-inheritable sclerotic bone dysplasia. We report a rare case of a 9 year old male with melorheostosis of carpal bones who presented with pain in right wrist joint on palmar flexion. Plain radiograph and CT performed revealed characteristic appearance of melorheostosis involving the scaphoid, lunate and trapezium.

CASE REPORT: A 9 Years old male child presented with complaints of persistent pain in right wrist joint over 1 month with limitation palmar flexion of the wrist.

On physical examination terminal palmar and dorsi flexion of the right wrist joint were restricted. Grip strength of right wrist is maintained. There was no significant limitation of daily activities.

Plain radiographs revealed hyperostosis of the scaphoid, lunate and trapezium involving both the cortex and medulla. There was no significant periosteal reaction. The carpal joint spaces were maintained. No obvious erosions / lytic lesions were noted. Computed tomography (CT) scans showed a high attenuation involving cortical hyperostosis.

Biochemical analysis including serum calcium, phosphorus, alkaline phosphatase, C-reactive protein and erythrocyte sedimentation rate revealed no significant abnormality.

A core biopsy was taken from the scaphoid and histopathological analysis revealed nonspecific, dense cortical bone replacing the trabecular system.

DISCUSSION: Melorheostosis is a rare non hereditary sclerosing bone dysplasia first described by the two French neurologists Leri and Joanny in $1922^{1}$.The origin of the word melorheostosis is from 
a combination of the Greek words 'melo' meaning 'limb', 'rheos' meaning 'flow' and 'osteon' meaning 'bone'2. This was in reference to the classic appearance on plain radiographs where the sclerosis appears to flow along the long axis of the bone, akin to candle wax dripping down one side of a candle.

Pathologically, this condition comprises of new bone formation on the periosteal and endosteal layers of the diaphyseal cortical bone. Typically, this is seen as irregular sclerosis along one side of a long bone. This condition most often affects the long bones of both the upper and lower extremities but may rarely affect small bones of the hands and feet or even the axial skeleton ${ }^{3}$.Within the hand, the short long bones like phalanges and metacarpals are usually involved and isolated involvement of the carpal bones is very rare.

The exact cause of this condition is yet unknown. However, several theories have been proposed including a genetic mutation involving the LEMD3 gene, an embryonal mesodermal disorder, a vascular abnormality and even infection of associated nerve roots ${ }^{4}$. The last theory was probably proposed to explain the common occurrence of this disorder in particular sclerotomes.

Several patterns of melorheostosis have been described including the monostotic (involving one bone), polyostotic (involving multiple bones) and the most common monomelic (involving one limb).Within a limb, melorheostosis is usually confined to a single sclerotome and even within a particular sclerotome, the lesions may cross the joint space to involve bones on either side but the intervening joint is usually not involved 5 .

Melorheostosis is often associated with various cutaneous manifestations overlying the involved bones including focal scleroderma, neurofibromatosis, various vascular and lymphatic malformations including telangiectasias. These skin changes usually parallel the bone involvement and are often restricted to the involved sclerotome ${ }^{6}$.

Clinically, melorheostosis is a chronic disorder with slow progression of symptoms. The usual presentation is pain, joint stiffness, and muscle contracture, limitation of motion, soft tissue abnormalities or progressive deformity ${ }^{7}$. It has no sex predilection and over $50 \%$ of the patients are diagnosed before the age of 20 years ${ }^{8}$.

The diagnosis of melorheostosis is usually based on classical imaging findings alone and a biopsy is usually not required. Also, histopathological analysis is most often not diagnostic.

On plain radiographs, there is increased bone density and hyperostosis is classically present along the cortical aspect of one side of bone resembling flowing candle wax - the 'dripping candle wax' sign'. In carpal, tarsal bones and in the epiphysis of long bones, the hyperostosis may be round or irregular as in our case. The CT appearance is similar to the typical undulating hyperostosis seen on radiographs ${ }^{10}$.These lesions appear well demarcated from the normal bone. On MR examination, sclerosis appears of low in signal intensity on both T1 and T2 weighted images. CT and MRI further help in delineating the soft tissue changes.

The radiological differential diagnosis include osteopoikilosis, osteopetrosis, arthrogryposis multiplex congenita, and osteopathia striata ${ }^{11}$. Radionuclide bone scan may be useful in differentiating melorheostosis from its mimics by demonstrating increased radiotracer uptake in the involved bones on blood pool as well as delayed phases in this condition ${ }^{12}$. When it affects the carpal bones, as in our case, the differential diagnosis would broaden to include other causes of carpal bone sclerosis including kienbock's disease, rheumatoid arthritis, chronic infections like tuberculosis, etc and often histopathology maybe required to rule out these conditions. 


\section{CASE REPORT}

Melorheostosis is a benign condition and usually does not need treatment. However, bisphosphonates have been reported to provide significant symptomatic relief by decreasing the bone turnover ${ }^{13}$.

CONCLUSION: Although melorheostosis is a relatively easy diagnosis to make radiologically when it involves long bones, it may be very difficult to diagnose involvement of small bones of the hands and feet. It is very important for radiologists and clinicians to be aware of this condition and its radiological appearance so as to make an early diagnosis and avoid unnecessary intervention.

\section{REFERENCES:}

1. Ethunandan M, Khosla N, Tilley E, Webb A. Bone dysplasia series. Melorheostosis: review and update. J Craniofac Surg. 2004; 15(6):1062-5. PMID: 15547407.

2. Van Durme CM, Starmans-Kool MJ, Peeters HR. Dripping candle wax. Neth J Med. 2012; 70(3):140-43. PMID: 22516580.

3. Greenspan A, Azouz EM. Bone dysplasia series. Melorheostosis: review and update. Can Assoc Radiol J. 1999; 50(5):324-30. PMID: 10555508.

4. Al Kaisii A, Skoumal M, Roetzer K, Grill F, Klaushofer K.A patient with melorheostosis manifesting with features similar to trichodento-osseous syndrome: a case report. J Med Case Rep. 2008; 2:51. PMID: 18284671.

5. Hai-Tao Long MD, Kang-Hua Li MD,Yong Zhu MD Severe Melorheostosis Involving the Ipsilateral Extremities Clin Orthop Relat Res 2009;467:2738-43.

6. Kim J, Cho SB, Cho S, Bang D. Unilateral nevoid telangiectasia associated with ipsilateral melorheostosis. Ann Dermatol. 2012; 24(2):206-8. PMID: 22577274.

7. Saadallaoui Ben Hamida K, Ksontini I, Rahali H, Mourali S, Fejraoui N, Bouhaouala H, Charfi MR, Dougui MH. Atypical form of melorheostosis improved by pamidronate. Tunis Med. 2009; 87(3):204-6. PMID: 19537015.

8. Masquijo JJ, Allende V. Melorheostosis of the hand in a pediatric patient. Arch Argent Pediatr. 2010; 108(6):e121-5. PMID: 21132236.

9. Bansal A. The Dripping Candle Wax Sign. Radiology 2008; 246(2):638-40. PMID: 18227553.

10. Motimaya AM, Meyers SP. Melorheostosis involving the cervical and upper thoracic spine: radiographic, CT, and MR imaging findings. AJNR Am J Neuroradiol. 2006; 27(6):1198-200. PMID: 16775263.

11. Schreck MA. Melorheostosis in a pediatric patient. J Podiatr Med Assoc. 2005;95(2):167-70. PMID: 15778476.

12. Izadyar S, Gholamrezanezhad A. Bone scintigraphy elucidates different metabolic stages of melorheostosis. Pan Afr Med J. 2012; 11:21. PMID: 22514755.

13. Hollick RJ, Black A, Reid D. Melorheostosis and its treatment with intravenous zoledronic acid. BMJ Case Rep. 2010; 2010. PMID: 22479293. 


\section{CASE REPORT}

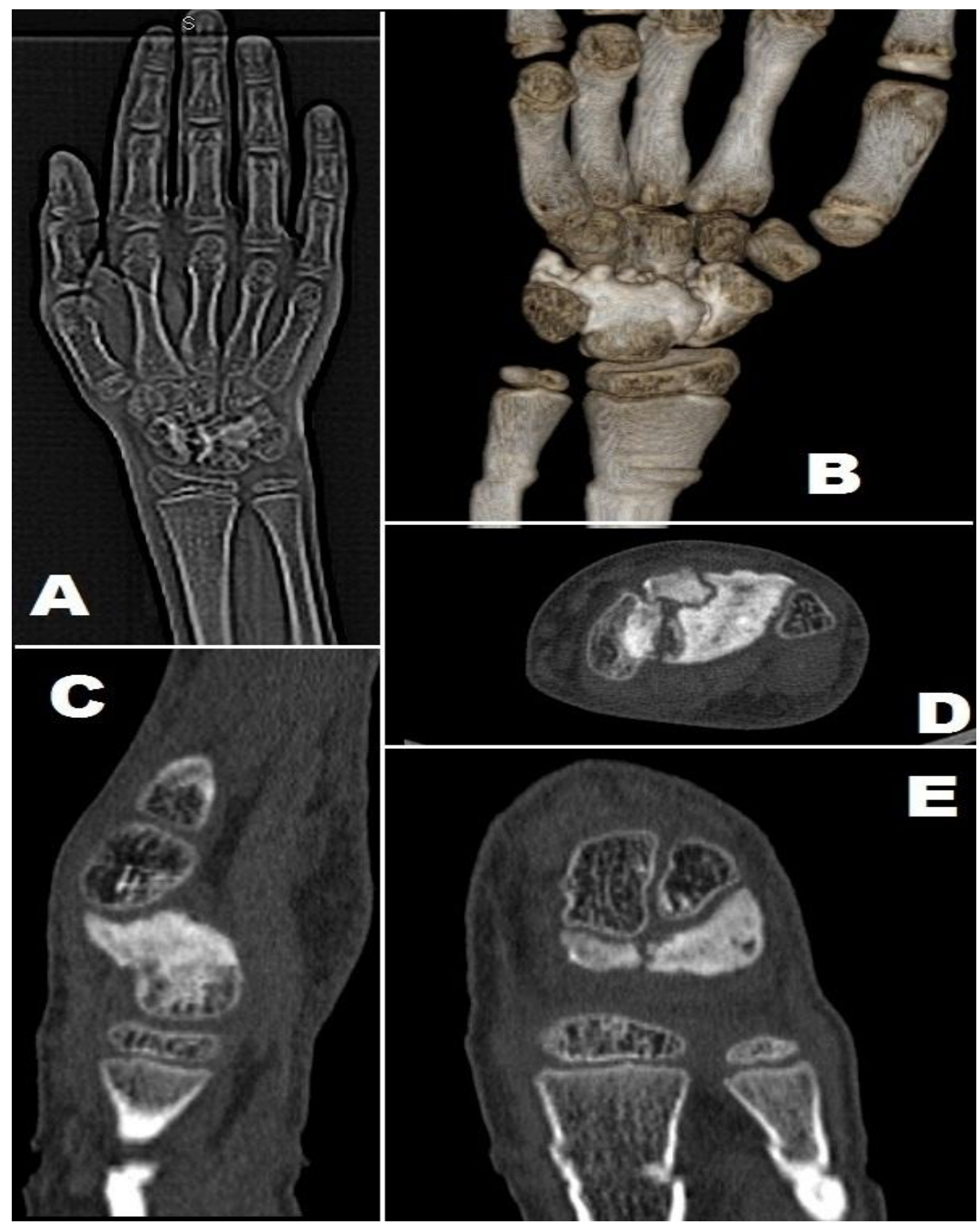

FIGURE LEGEND: CT Scan of right wrist joint of 9 year old male child showing hyperostosis of the scaphoid, lunate and trapezium. (A) Scout view, (B) Volume Rendering Technique, (C) CT sagittal view, (D) CT axial view and (E) CT coronal view. 